\title{
On the Distributed Order Fractional Multi-Strain Tuberculosis Model: A Numerical Study
}

\author{
N. H. Sweilam ${ }^{1, *}$, S. M. AL-Mekhlafi ${ }^{2}$, A. O. Albalawi ${ }^{3}$ \\ ${ }^{1}$ Department of Mathematics, Faculty of Science, Cairo University, Giza, Egypt \\ ${ }^{2}$ Department of Mathematics, Faculty of Education, Sana'a University, Yemen \\ ${ }^{3}$ Department of Mathematics, Faculty of Science, Shaqra University, Riyadh, KSA
}

\begin{abstract}
In this paper, a novel mathematical distributed order fractional model of multi-strain Tuberculosis is presented. The proposed model is governed by a system of distributed order fractional differential equations, where the distributed order fractional derivative is defined in the sense of the Grünwald-Letinkov definition. A nonstandard finite difference method is proposed to study the resulting system . The stability analysis of the proposed model is discussed. Numerical simulations show that, the nonstandard finite difference method can be applied to solve such distributed order fractional differential equations simply and effectively.
\end{abstract}

Keywords Tuberculosis; Distributed order fractional calculus; Grünwald-Letnikov definition; Nonstandard finite difference method.

AMS 2010 subject classifications 65M06; 65M12; 26A33

DOI: $10.19139 /$ soic-2310-5070-621

\section{Introduction}

In fact, the fractional calculus has been acknowledged as a promising mathematical tool to efficiently characterize the historical memory and global correlation of complex dynamic systems, phenomena or structures. However, various literature indicated that the memory and/or nonlocality of the system may change with time, space or other conditions [1], [2]. The variable-order (VO) fractional operators depending on their non-stationary powerlaw kernel can describe the memory and hereditary properties of many physical phenomena and processes [3], [4].

It is known that the distributed order derivatives are fractional order derivatives that have been integrated over the order of the derivative within a given range. The idea of fractional derivative of distributed order is stated by [18] and later developed by Caputo himself in [19, 20] and Bagley and Torvik [21]. Many researchers used this idea, they applied it to some interesting mathematical models of partial fractional differential equation of distributed order. Diethelm and Ford [22] used a numerical technique along with its error analysis to solve the distributed order differential equation and analyze the physical phenomena and engineering problems; see [22] and the references cited therein. Recently Saberi Najafi et al. [23, 24] studied the stability analysis of distributed order fractional differential equations with respect to the nonnegative density function.

Mathematical models can project how infectious diseases progress, to show the likely outcome of an epidemic, and help inform public health interventions. In epidemiology, compartmental models serve as the base mathematical framework for understanding the complex dynamics of these systems [5].

\footnotetext{
*Correspondence to: Department of Mathematics, Faculty of Science, Cairo University, Giza, Egypt. E-mail: nsweilam@ @sci.cu.edu.eg, smdk100@yahoo.com
}

ISSN 2310-5070 (online) ISSN 2311-004X (print)

Copyright (C) 2020 International Academic Press 
The multi-strain TB model which incorporates three strains: drug-sensitive, emerging multi-drug resistant(MDR) and extensively drug-resistant(XDR ) is developed by Arino and Soliman [6] in (2015). This model included several factors of spreading TB such as the fast infection, the exogenous reinfection and secondary infection along with the resistance factor. Sweilam and AL-Mekhlafi introduced some numerical studies for this model in ([25]-[32]).

In this paper, we will generalize the integer order multi-strain Tuberculosis model [30] to the distributed order fractional domain. Nonstandard finite difference method (NSFDM) is introduced to solve this system. The obtained results by the proposed method are compared with the obtained results by the standard finite difference method (SFDM).

This paper is organized as follows: In section 2, mathematical preliminaries of the fractional and distributed order fractional calculus theory which are required for establishing the results are given. In section 3 , the proposed model of distributed order fractional derivatives and the stability of distributed order fractional of the proposed model are discussed. In section 4,NSFD for distributed order fractional differential equations is given. Numerical simulations are given in section 5. In section 6, the conclusions are given.

\section{Elementary Definitions}

In the following, some basic definitions and properties in the theory of the distributed order fractional calculus are presented. Moreover, we introduce the main aspects concerning nonstandard discretization methods. There are many definitions of fractional derivatives of order $\alpha>0[10,15]$ such as Grünwald-Letinkov's definition (GL), Riemann-Liouville's definition (RL), and Caputo's fractional derivative. The RL definition is given as:

$$
{ }^{R L} D_{t}^{\alpha} z(t)=\frac{1}{\Gamma(n-\alpha)} \frac{d^{n}}{d t^{n}} \int_{0}^{t}(t-\tau)^{(n-\alpha-1)} z(\tau) d \tau,
$$

where $n$ is the first integer which is not less than $\alpha$, that is, $n-1<\alpha<n$ and $\Gamma(\cdot)$ is a Gamma function. The Caputo fractional derivative of $z(t)$ is defined as

$$
{ }^{C} D_{t}^{\alpha} z(t)=\frac{1}{\Gamma(n-\alpha)} \int_{0}^{t}(t-\tau)^{(n-\alpha-1)} z^{(n)}(\tau) d \tau .
$$

It is known that Grünwald-Letinkov's definition and Riemann-Liouville definition are equivalence, but the Grünwald-Letinkov's definition is very easily utilized for the numerical evaluations see $[8,11]$. Hence, in order to apply nonstandard finite difference schemes laters, we have chosen Grünwald-Letinkov's approximation formula for the distributed order fractional derivative [11]:

$$
{ }^{G L} D_{t}^{\alpha} z(t)=\lim _{h \rightarrow 0} h^{-\alpha} \sum_{r=0}^{m}(-1)^{r}\left(\begin{array}{c}
\alpha \\
r
\end{array}\right) z(t-r h),
$$

where, $m=\left[\frac{x}{h}\right]$ denotes the integer part of $\frac{x}{h}$ and $h$ is the step-size. Equation 3 can be discretized as follows:

$$
\sum_{r=0}^{m} \omega_{r}^{\alpha} z\left(t_{m-r}\right)=g\left(t_{m}, z\left(x_{m}\right)\right) \quad m=1,2,3, \cdots
$$

Where $g\left(t_{m}, z\left(x_{m}\right)\right)={ }^{C} D_{t}^{\alpha} z(t), x_{m}=m h$, and $\omega_{r}^{\alpha}$, are the Grünwald-Letinkov coefficients define as follows:

$$
\omega_{r}^{\alpha}=\left(1-\frac{1+\alpha}{r}\right) \omega_{r-1}^{\alpha}, \quad \omega_{0}^{\alpha}=h^{-\alpha}, \quad r=1,2,3, \cdots .
$$

Remark 1

The approximate Grünwald-Letinkov definition is given as follows:

$$
{ }^{C} D_{t}^{\alpha} z(t) \approx \sum_{r=0}^{m} \omega_{r}^{\alpha} z\left(t_{m-r}\right) \quad m=1,2,3, \cdots
$$

where the step size of $h$ is assumed to be very small $[9,15,17]$. 
Definition 2.1

[20] For $q(\alpha \geq 0), q \neq 0, \alpha \in(0,1]$ and $\int_{0}^{1} q(\alpha) d \alpha=c_{0}>0$, the left and right sided fractional derivatives of distributed orders in the Riemann-Liouville sense are defined, respectively, by:

$$
\begin{aligned}
& { }_{a}^{R L} D_{t}^{q(\alpha)} f(t)=\int_{0}^{1} q(\alpha){ }_{a}^{R L} D_{t}^{\alpha} f(t) d \alpha, \\
& { }_{t}^{R L} D_{b}^{q(\alpha)} f(t)=\int_{0}^{1} q(\alpha){ }_{t}^{R L} D_{b}^{\alpha} f(t) d \alpha .
\end{aligned}
$$

\section{Definition 2.2}

[20] For $q(0 \leq \alpha), q \neq 0, \alpha \in(0,1]$ and $\int_{0}^{1} q(\alpha) d \alpha=c_{0}>0$, the left and right sided fractional derivatives of distributed orders in the Caputo sense are defined, respectively, by:

$$
\begin{aligned}
& { }_{a}^{C} D_{t}^{q(\alpha)} f(t)=\int_{0}^{1} q(\alpha){ }_{a}^{C} D_{t}^{\alpha} f(t) d \alpha, \\
& { }_{t}^{C} D_{b}^{q(\alpha)} f(t)=\int_{0}^{1} q(\alpha){ }_{t}^{C} D_{b}^{\alpha} f(t) d \alpha .
\end{aligned}
$$

\subsection{NSFD Discretization}

It is known that, the numerical scheme is called nonstandard method if at least one of the following conditions are satisfied [30]:

1. The nonlocal approximation is used.

2. The discretization of the derivative is not traditional and uses a nonnegative function ([12], [14]).

For more details on the nonstandard method see [16] and the references cited therein.

\section{Remark 2}

Almost all of standard procedures yield schemes which are convergent with restriction of step size while NSFDM are convergent for any step size. Also, in addition to the usual properties of consistency, stability and hence convergence for nonstandard finite difference schemes, they produce numerical solutions which also exhibit essential properties of the solution, for more details see ([12]-[14], [16]).

\section{Distributed Order Fractional Multi-Strain TB Model}

In this section, the fractional multi-strain TB model is modified by integrating over all possible orders of the fractional time derivative. This is called a distributed order derivative. The population of interest is divided into eight compartments depending on their epidemiological stages as follows: susceptible $(S)$; latently infected with drug sensitive TB $\left(L_{s}\right)$; latently infected with MDR TB $\left(L_{m}\right)$; latently infected with XDR TB $\left(L_{x}\right)$; sensitive drug TB infectious $\left(I_{s}\right)$; MDR TB infectious $\left(I_{m}\right)$; XDR TB infectious $\left(I_{x}\right)$; recovered $R$. All interpretation and meaning of parameters for this model see [28]. One of the main assumptions of this model is that, the total population $N(t)$, with $N(t)=S(t)+L_{s}(t)+L_{m}(t)+L_{x}(t)+I_{s}(t)+I_{m}(t)+I_{x}(t)+R(t)$ is variable of the time. The new 
system is described by distributed order fractional derivatives as follows:

$$
\begin{aligned}
&{ }_{0}^{C} D_{t}^{q(\alpha)} S= b-d S-\beta_{s} \frac{S I_{s}}{N}-\beta_{m} \frac{S I_{m}}{N}-\beta_{x} \frac{S I_{x}}{N}, \\
&{ }_{0}^{C} D_{t}^{q(\alpha)} L_{s}= \lambda_{s} \beta_{s} \frac{S I_{s}}{N}+\sigma_{s} \lambda_{s} \beta_{s} \frac{R I_{s}}{N}+\gamma_{s} I_{s}-\alpha_{s s} \beta_{s} \frac{L_{s} I_{s}}{N}-\alpha_{s m} \beta_{m} \frac{L_{s} I_{m}}{N}-\alpha_{s x} \beta_{x} \frac{L_{s} I_{x}}{N} \\
&-\left(d+\varepsilon_{s}+t_{1 s}\right) L_{s}, \\
&{ }_{0}^{C} D_{t}^{q(\alpha)} L_{m}= \lambda_{m} \beta_{m} \frac{S I_{m}}{N}+\sigma_{m} \lambda_{m} \beta_{m} \frac{R I_{m}}{N}+\gamma_{m} I_{m}+\alpha_{s m} \beta_{m} \lambda_{m} \frac{L_{s} I_{m}}{N}+\left(1-P_{1}\right) t_{1 s} L_{s} \\
&+\left(1-P_{2}\right) t_{2 s} I_{s}-\alpha_{m m} \beta_{m} \frac{L_{m} I_{m}}{N}-\alpha_{m x} \beta_{x} \frac{L_{m} I_{x}}{N}-\left(d+\varepsilon_{m}\right) L_{m}, \\
&+\left(1-P_{3}\right) t_{2 m} I_{m}-\alpha_{x x} \beta_{x} \frac{L_{x} I_{x}}{N}-\left(d+\varepsilon_{x}\right) L_{x}, \\
&{ }_{0}^{C} D_{t}^{q(\alpha)} L_{x}= \lambda_{x} \beta_{x} \frac{S I_{x}}{N}+\sigma_{x} \lambda_{x} \beta_{x} \frac{R I_{x}}{N}+\gamma_{x} I_{x}+\alpha_{s x} \beta_{x} \lambda_{x} \frac{L_{s} I_{x}}{N}+\alpha_{m x} \beta_{x} \lambda_{x} \frac{L_{m} I_{x}}{N} \\
&+{ }_{0}^{C} D_{t}^{q(\alpha)} I_{s}= \\
& \alpha_{s s} \beta_{s} \frac{L_{s} I_{s}}{N}+\left(1-\lambda_{s}\right) \beta_{s}\left(\frac{S I_{s}}{N}+\sigma_{s} \frac{R I_{s}}{N}\right)+\varepsilon_{s} L_{s}-\left(d+\delta_{s}+t_{2 s}+\gamma_{s}\right) I_{s}, \\
&{ }_{0}^{C} D_{t}^{q(\alpha)} I_{m}= \alpha_{m m} \beta_{m} \frac{L_{m} I_{m}}{N}+\left(1-\lambda_{m}\right) \beta_{m}\left(\frac{S I_{m}}{N}+\sigma_{m} \frac{R I_{m}}{N}+\alpha_{s m} \frac{L_{s} I_{m}}{N}\right)+\varepsilon_{m} L_{m} \\
&-\left(d+\delta_{m}+t_{2 m}+\gamma_{m}\right) I_{m}, \\
&-d R . \\
&-\left(d+\delta_{x}^{C} D_{t}^{q(\alpha)} I_{x}=\alpha_{x x} \beta_{x} \frac{L_{x} I_{x}}{N}+\left(1-t_{2 x}\right) \beta_{x}\left(\frac{S I_{x}}{N}+\gamma_{x}\right) I_{x},\right. \\
&{ }_{0}^{C} D_{t}^{q(\alpha)} R= P_{1} t_{1 s} L_{s}+P_{2} t_{2 s} I_{s}+P_{3} t_{2 m} I_{m}+t_{2 x} I_{x}-\sigma_{s} \frac{L_{s} I_{x}}{N}+\alpha_{m x} \frac{R I_{s}}{N}-\sigma_{m} \beta_{m} \frac{R I_{m}}{N}-\sigma_{x} \beta_{x} \frac{R I_{x}}{N} \\
&-d R L_{x}
\end{aligned}
$$

\section{Remark 3}

In special case, if $q(\alpha)=\delta(\alpha-\xi), 0<\xi \leq 1$, where, $\delta(\alpha)$ is the Dirac delta function, then, we claim the fractional order multi-strain TB model of order $\xi$.

\subsection{Stability analysis of distributed order fractional systems}

The linear distributed order fractional systems can be expressed as:

$$
\begin{aligned}
& { }_{0}^{C} D_{t}^{q(\alpha)} Y=B Y(t), \\
& Y(0)=Y_{0},
\end{aligned}
$$

where, $Y(t)=\left(S(t), L_{s}(t), L_{m}(t), L_{x}(t), I_{s}(t), I_{m}(t), I_{x}(t), R(t)\right) \in R^{8}$, the matrix $B \in R^{n \times n}$ and $q(\alpha)$, the density function, $0<\alpha \leq 1$. Saberi Najafi et al. [24] have obtained the general solution of the distributed order fractional systems (18), as follows:

$$
\begin{aligned}
Y(t) & =Y(0)+\frac{1}{\pi} \int_{0}^{t} \int_{0}^{\infty} \int_{0}^{\infty} e^{-r t+B \tau-\rho \cos (\pi \gamma)} \\
& \times \sin (\rho \sin (\pi \gamma)) \sin (B Y(0)) d r d \tau d t
\end{aligned}
$$

where, $\rho=|A(s)|, \gamma=\frac{1}{\pi} \arg [A(s)], r=e^{i \pi}$ and $A(s)=\rho \cos (\pi \gamma)+i \rho \sin (\pi \gamma)$. Now, we will recall some theorems and definitions on linear distributed order fractional equations and then we will show that theorem for nonlinear distributed order fractional equations as well.

Theorem 3.1

[24] The distributed order fractional system of (18) is asymptotically stable if and only if all roots of $\operatorname{det}(A(s) I-$ $B)=0$, have negative real parts.

\section{Remark 4}

The value of $\operatorname{det}(A(s) I-B)=0$, is the characteristic function of the matrix $B$ with respect to the distributed 
function $A(s)$, where $\int_{0}^{1} q(\alpha) s^{\alpha} d \alpha$ is the distributed function with respect to the density function, where, $A(s)=s^{\alpha}$. Also, $\operatorname{det}(A(s) I-B)=0$, is reduced to $s^{\alpha} I-B=0$. Let $\lambda=s^{\alpha}$, then $s=\lambda^{\frac{1}{\alpha}}$, and we have $\left|\arg \lambda^{\frac{1}{\alpha}}\right|>\frac{\pi}{2}$. Thus, all the roots $\lambda$ of equation $\operatorname{det}(\lambda I-B)=0$, satisfy $\left|\arg \lambda^{\frac{1}{\alpha}}\right|>\alpha \frac{\pi}{2}$.

\section{Definition 3.1}

The inertia of the system (18) is the triple:

$$
\left(\Theta_{n A(s)} B, \vartheta_{n A(s)} B, \Phi_{n A(s)} B\right)=\Upsilon_{n A(s)} B,
$$

where, $\Theta_{n A(s)} B, \vartheta_{n A(s)} B$ and $\Phi_{n A(s)} B$, are, respectively, the number of roots of $\operatorname{det}(A(s) I-A)=0$, with positive, negative, and zero real parts, where $A(s)=\left(A_{1}(s), A_{2}(s), \ldots, A_{n}(s)\right)^{T}$ is the distributed function with respect to the density function $q(\alpha)$.

Theorem 3.2

[24] The linear distributed order fractional system (18) is asymptotically stable if and only if any of the following equivalent conditions holds:

1. $\Theta_{n A(s)}(B)=\Phi_{n A(s)} B=0$,

2. all roots $s$ of the characteristic function of $B$ with respect to $A(s)=\left(A_{1}(s), A_{2}(s), \ldots, A_{n}(s)\right)^{T}$ satisfy $|\arg (s)|>\frac{\pi}{2}$.

Next, we will mainly discuss the stability of a nonlinear autonomous distributed order fractional system, which can be described by

$$
\begin{aligned}
& { }_{0}^{C} D_{t}^{q(\alpha)} Y=G(Y(t)), \\
& Y(0)=Y_{0},
\end{aligned}
$$

with the initial value $Y(0)=Y_{0}$, where,

$$
G(Y(t))=\left(\begin{array}{c}
g_{1}\left(y_{1}(t), y_{2}(t), \ldots, y_{N}(t)\right) \\
g_{2}\left(y_{1}(t), y_{2}(t), \ldots, y_{N}(t)\right) \\
\cdot \\
\cdot \\
\cdot \\
g_{n}\left(y_{1}(t), y_{2}(t), \ldots, y_{N}(t)\right)
\end{array}\right)
$$

Theorem 3.3

Let $Y^{*}=\left(y_{1}^{*}, y_{2}^{*}, \ldots, y_{n}^{*}\right)^{T}$ be the equilibrium of system 20 ; that is, ${ }_{0}^{C} D_{t}^{q(\alpha)} Y^{*}=G\left(Y^{*}\right)=0$ and $J=\left.\left(\frac{\partial G}{\partial Y}\right)\right|_{Y=Y^{*}}$ is the Jacobian matrix at the point $Y^{*}$ then the point $Y^{*}$ is asymptotically stable if and only if all roots $s$ of the characteristic function of $J$ with respect to $A(s)=\left(A_{1}(s), A_{2}(s), \ldots, A_{n}(s)\right)^{T}$ satisfy $|\arg (s)|>\frac{\pi}{2}$.

Proof

Let $\varrho(t)=Y(t)-Y^{*}(t)$, where $\varrho(t)$ is a small disturbance from a fixed point. Therefore

$$
{ }_{0}^{C} D_{t}^{q(\alpha)} \varrho(t)={ }_{0}^{C} D_{t}^{q(\alpha)}\left(Y(t)-Y^{*}\right) \text {, }
$$

since, ${ }_{0}^{C} D_{t}^{q(\alpha)}\left(Y(t)-Y^{*}\right)={ }_{0}^{C} D_{t}^{q(\alpha)} Y(t)-{ }_{0}^{C} D_{t}^{q(\alpha)} Y^{*}=0$; thus, we have

$$
\begin{aligned}
{ }_{0}^{C} D_{t}^{q(\alpha)} \varrho(t) & ={ }_{0}^{C} D_{t}^{q(\alpha)} Y(t)=G(Y(t))=G\left(\varrho(t)+Y^{*}\right), \\
& =G\left(Y^{*}\right)+J \varrho(t)+\text { higher order terms } \approx J \varrho(t) .
\end{aligned}
$$

System (21) can be written as

$$
{ }_{0}^{C} D_{t}^{q(\alpha)} \varrho(t)=J \varrho(t),
$$

with the initial value $\varrho(0)=Y_{0}-Y^{*}$. The analytical procedure of linearization is based on the fact that if the matrix $J$ has no purely imaginary eigenvalues, then the trajectories of the nonlinear system in the neighborhood of the equilibrium point have the same form as the trajectories of the linear system (23). Hence, by applying Theorem 3.2 , the linear system (23) is asymptotically stable if and only if all roots $s$ of the characteristic function of $J$ with respect to $A(s)=\left(A_{1}(s), A_{2}(s), \ldots, A_{n}(s)\right)^{T}$ satisfy $|\arg (s)|>\frac{\pi}{2}$, which implies that the equilibrium $Y^{*}$ of the nonlinear distributed order fractional system $(20)$ is as asymptotically stable. 


\section{Remark 5}

The nonlinear distributed order fractional system (20) in the point $Y^{*}$ is asymptotically stable if and only if $\Theta_{n A(s)} B=\Phi_{n A(s)} B=0$.

Table 1. All parameters in the system (10) - (17) and the reference of the parameters.

\begin{tabular}{|c|c|c|}
\hline parameter & value & Reference \\
\hline$b$ & 3190 & Assumed \\
\hline$d$ & 0.38 & {$[33]$} \\
\hline$\beta_{s}=\beta_{m}=\beta_{x}$ & 14 & {$[33]$} \\
\hline$\lambda_{s}=\lambda_{m}=\lambda_{x}$ & 0.5 & Assumed \\
\hline$\varepsilon_{s}=\varepsilon_{m}=\varepsilon_{x}$ & 0.5 & Assumed \\
\hline$\alpha_{r 1, r 2}$ & 0.05 & Assumed \\
\hline$\gamma_{s}=\gamma_{m}=\gamma_{x}$ & 0.3 & Assumed \\
\hline$t_{1 s}$ & 0.88 & {$[33]$} \\
\hline$t_{2 r}: r \in(s, m, x)$ & $t_{2 s}=0.88 ; t_{2 m}=t_{2 x}=0.034$ & {$[33]$} \\
\hline$\sigma_{r}$ & 0.25 & {$[33]$} \\
\hline$P_{r}$ & 0.88 & {$[33]$} \\
\hline$\delta_{r}$ & 0.045 & {$[33]$} \\
\hline
\end{tabular}

\section{NSFD for Distributed Order Fractional Differential Equations}

NSFD schemes were introduced by Mickens in the 1980s as a powerful numerical method that preserves significant properties of exact solutions of the involved differential equation [7]. We defined distributed order fractional derivative using Grünwald-Letinkov's sense and applying NSFD. Then by using the midpoint quadrature rule. The system $10-17$ can be discretized as follows:

$$
\begin{aligned}
\sum_{i=1}^{K} \frac{q\left(\alpha_{i}\right)}{K} \sum_{j=0}^{n+1} \omega_{j}^{\alpha_{i}} S^{n+1-j}= & b-d S^{n+1}-\beta_{s} \frac{S^{n+1} I_{s}^{n}}{N^{n}}-\beta_{m} \frac{S^{n+1} I_{m}^{n}}{N^{n}}-\beta_{x} \frac{S^{n+1} I_{x}^{n}}{N^{n}} \\
\sum_{i=1}^{K} \frac{q\left(\alpha_{i}\right)}{K} \sum_{j=0}^{n+1} \omega_{j}^{\alpha_{i}} L_{s}^{n+1-j}= & \lambda_{s} \beta_{s} \frac{S^{n+1} I_{s}^{n}}{N^{n}}+\sigma_{s} \lambda_{s} \beta_{s} \frac{R^{n+1} I_{s}^{n}}{N^{n}}+\gamma_{s} I_{s}^{n}-\alpha_{s s} \beta_{s} \frac{L_{s}^{n+1} I_{s}^{n}}{N^{n}}-\alpha_{s x} \beta_{x} \frac{L_{s}^{n+1} I_{x}^{n}}{N^{n}} \\
& -\left(d+\varepsilon_{s}+t_{1 s}\right) L_{s}^{n+1}-\alpha_{s m} \beta_{m} \frac{L_{s}^{n+1} I_{m}^{n}}{N^{n}}, \\
\sum_{i=1}^{K} \frac{q\left(\alpha_{i}\right)}{K} \sum_{j=0}^{n+1} \omega_{j}^{\alpha_{i}} L_{m}^{n+1-j}= & \lambda_{m} \beta_{m} \frac{S^{n+1} I_{m}^{n}}{N^{n}}+\sigma_{m} \lambda_{m} \beta_{m} \frac{R^{n+1} I_{m}^{n}}{N^{n}}+\lambda_{m} \alpha_{s m} \beta_{m} \frac{L_{s}^{n+1} I_{m}^{n}}{N^{n}}+\gamma_{m} I_{m}^{n} \\
& +t_{1 s} L_{s}^{n+1}-P_{1} t_{1 s} L_{s}^{n+1}+t_{2 s} I_{s}^{n}-P_{2} t_{2 s} I_{s}^{n}-\alpha_{m m} \beta_{m} \frac{L_{m}^{n+1} I_{m}^{n}}{N^{n}} \\
& -\alpha_{m x} \beta_{x} \frac{L_{m}^{n+1} I_{x}^{n}}{N^{n}}-\left(d+\varepsilon_{m}\right) L_{m}^{n+1},
\end{aligned}
$$




$$
\begin{aligned}
& \sum_{i=1}^{K} \frac{q\left(\alpha_{i}\right)}{K} \sum_{j=0}^{n+1} \omega_{j}^{\alpha_{i}} L_{x}^{n+1-j}=\lambda_{x} \beta_{x} \frac{S^{n+1} I_{x}^{n}}{N^{n}}+\sigma_{x} \lambda_{x} \beta_{x} \frac{R^{n+1} I_{x}^{n}}{N^{n}}+\lambda_{x} \alpha_{s x} \beta_{x} \frac{L_{s}^{n+1} I_{x}^{n}}{N^{n}}+\gamma_{x} I_{x}^{n} \\
& +\lambda_{x} \alpha_{m x} \beta_{x} \frac{L_{m}^{n+1} I_{x}^{n}}{N^{n}}+t_{2 m} I_{m}^{n}-P_{3} t_{2 m} I_{m}^{n}-\alpha_{x x} \beta_{x} \frac{L_{x}^{n+1} I_{x}^{n}}{N^{n}} \\
& -\left(d+\varepsilon_{x}\right) L_{x}^{n+1}, \\
& \sum_{i=1}^{K} \frac{q\left(\alpha_{i}\right)}{K} \sum_{j=0}^{n+1} \omega_{j}^{\alpha_{i}} I_{s}^{n+1-j}=\alpha_{s s} \beta_{s} \frac{L_{s}^{n+1} I_{s}^{n}}{N^{n}}+\left(1-\lambda_{s}\right) \beta_{s}\left(\frac{S^{n+1} I_{s}^{n}}{N^{n}}+\sigma_{s} \frac{R^{n+1} I_{s}^{n}}{N^{n}}\right)+\varepsilon_{s} L_{s}^{n+1} \\
& -\left(d+\delta_{s}\right) I_{s}^{n+1}-\left(\gamma_{s}+t_{2 s}\right) I_{s}^{n}, \\
& \sum_{i=1}^{K} \frac{q\left(\alpha_{i}\right)}{K} \sum_{j=0}^{n+1} \omega_{j}^{\alpha_{i}} I_{m}^{n+1-j}=\alpha_{m m} \beta_{m} \frac{L_{m}^{n+1} I_{m}^{n}}{N^{n}}+\left(1-\lambda_{m}\right) \beta_{m}\left(\frac{S^{n+1} I_{m}^{n}}{N^{n}}+\sigma_{m} \frac{R^{n+1} I_{m}^{n}}{N^{n}}+\alpha_{s m} \frac{L_{s}^{n+1} I_{m}^{n}}{N^{n}}\right) \\
& +\varepsilon_{m} L_{m}^{n+1}-\left(d+\delta_{m}\right) I_{m}^{n+1}-\left(\gamma_{s}+t_{2 m}\right) I_{m}^{n}, \\
& \sum_{i=1}^{K} \frac{q\left(\alpha_{i}\right)}{K} \sum_{j=0}^{n+1} \omega_{j}^{\alpha_{i}} I_{x}^{n+1-j}=\alpha_{x x} \beta_{x} \frac{L_{x}^{n+1} I_{x}^{n}}{N^{n}}+\left(1-\lambda_{x}\right) \beta_{m}\left(\frac{S^{n+1} I_{x}^{n}}{N^{n}}+\sigma_{x} \frac{R^{n+1} I_{x}^{n}}{N^{n}}+\alpha_{m x} \frac{L_{x}^{n+1} I_{m}^{n}}{N^{n}}\right) \\
& +\varepsilon_{x} L_{x}^{n+1}-\left(d+\delta_{x}\right) I_{x}^{n+1}-\left(\gamma_{x}+t_{2 x}\right) I_{x}^{n}, \\
& \sum_{i=1}^{K} \frac{q\left(\alpha_{i}\right)}{K} \sum_{j=0}^{n+1} \omega_{j}^{q\left(\alpha_{i}\right)} R^{n+1-j}=P_{1} t_{1 s} L_{s}^{n+1}+P_{2} t_{2 s} I_{s}^{n}+P_{3} t_{2 m} I_{m}^{n}+t_{2 x} I_{x}^{n}-d R^{n+1}-\sigma_{s} \beta_{s} \frac{R^{n+1} I_{s}^{n}}{N^{n}} \\
& -\sigma_{m} \beta_{m} \frac{R^{n+1} I_{m}^{n}}{N^{n}}-\sigma_{x} \beta_{x} \frac{R^{n+1} I_{x}^{n}}{N^{n}} .
\end{aligned}
$$

The discretizations for $N(t)$ is given as:

$$
N^{n}=S^{n}+L_{s}^{n}+L_{m}^{n}+L_{x}^{n}+I_{s}^{n}+I_{m}^{n}+I_{x}^{n}+R^{n} .
$$

Where, $\omega_{0}^{\alpha_{i}}=\left(\varphi_{s}(h)\right)^{-\alpha_{i}}, s=1,2, \cdots, 8$ and $0<\alpha_{i}<1$. The nonlocal approximations are used for the nonlinear terms and the following denominator functions are used:

Then, we can obtain

$$
\begin{aligned}
& \varphi_{1}(h)=\frac{e^{d h}-1}{d}, \quad \varphi_{2}(h)=\frac{e^{\left(d+\varepsilon_{s}+t_{1 s}\right) h}-1}{\left(d+\varepsilon_{s}+t_{1 s}\right)}, \\
& \varphi_{3}(h)=\frac{e^{\left(d+\varepsilon_{m}\right) h}-1}{\left(d+\varepsilon_{m}\right)}, \quad \varphi_{4}(h)=\frac{e^{\left(d+\varepsilon_{x}\right) h}-1}{\left(d+\varepsilon_{x}\right)}, \\
& \varphi_{5}(h)=\frac{1-e^{-\left(d+\delta_{s}\right) h}}{\left(\gamma_{s}+t_{2 s}\right)}, \quad \varphi_{6}(h)=\frac{1-e^{-\left(d+\delta_{m}\right) h}}{\left(\gamma_{m}+t_{2 m}\right)}, \\
& \varphi_{7}(h)=\frac{1-e^{-\left(d+\delta_{x}\right) h}}{\left(\gamma_{x}+t_{2 x}\right)}, \quad \varphi_{8}(h)=\frac{e^{d h}-1}{d} .
\end{aligned}
$$

$$
\begin{aligned}
S^{n+1}= & \frac{b-\sum_{i=1}^{K} \frac{q\left(\alpha_{i}\right)}{K} \sum_{j=1}^{n+1} \omega_{j}^{\alpha_{i}} S^{n+1-j}}{\sum_{i=1}^{K} \frac{q\left(\alpha_{i}\right)}{K}\left(\varphi_{1}(h)\right)^{-\alpha_{i}}+d+\frac{\beta_{s} I_{s}^{n}+\beta_{m} I_{m}^{n}+\beta_{x} I_{x}^{n}}{N^{n}}} \\
L_{s}^{n+1}= & \frac{\frac{\beta_{s} I_{s}^{n}}{N^{n}} \lambda_{s}\left(S^{n+1}+\sigma_{s} R^{n+1}\right)+\gamma_{s} I_{s}^{n}-\sum_{i=1}^{K} \frac{q\left(\alpha_{i}\right)}{K} \sum_{j=1}^{n+1} \omega_{j}^{\alpha} L_{s}^{n+1-j}}{\sum_{i=1}^{K} \frac{q\left(\alpha_{i}\right)}{K} q\left(\alpha_{i}\right)\left(\varphi_{2}(h)\right)^{-\alpha_{i}}+\left(d+t_{1 s}+\varepsilon_{s}\right)+\frac{1}{N^{n}}\left(\alpha_{s s} \beta_{s} I_{s}^{n}+\alpha_{s m} \beta_{m} I_{m}^{n}+\alpha_{s x} \beta_{x} I_{x}\right)}, \\
L_{m}^{n+1}= & \frac{\frac{\beta_{m} \lambda_{m} I_{m}^{n}}{N^{n}}\left(S^{n+1}+\sigma_{m} R^{n+1}+\alpha_{s m} L_{s}^{n+1}\right)+\gamma_{m} I_{m}^{n}+t_{1 s} L_{s}^{n+1}\left(1-P_{1}\right)}{\sum_{i=1}^{K} \frac{q\left(\alpha_{i}\right)}{K} q\left(\alpha_{i}\right)\left(\varphi_{3}(h)\right)^{-\alpha_{i}}+\left(d+\varepsilon_{m}\right)+\frac{1}{N^{n}}\left(\alpha_{m m} \beta_{m} I_{m}^{n}+\alpha_{m x} \beta_{x} I_{x}^{n}\right)} \\
& +\frac{t_{2 s} I_{s}^{n}\left(1-P_{2}\right)-\sum_{i=1}^{K} \frac{q\left(\alpha_{i}\right)}{K} \sum_{j=1}^{n+1} \omega_{j}^{\alpha_{i}} L_{m}^{n+1-j}}{\sum_{i=1}^{K} \frac{q\left(\alpha_{i}\right)}{K}\left(\varphi_{3}(h)\right)^{-\alpha_{i}}+\left(d+\varepsilon_{m}\right)+\frac{1}{N^{n}}\left(\alpha_{m m} \beta_{m} I_{m}^{n}+\alpha_{m x} \beta_{x} I_{x}^{n}\right)},
\end{aligned}
$$




$$
\begin{aligned}
L_{x}^{n+1}= & \frac{\frac{\beta_{x} \lambda_{x} I_{x}^{n}}{N^{n}}\left(S^{n+1}+\sigma_{x} R^{n+1}+\alpha_{s x} L_{s}^{n+1}+\alpha_{m x} L_{m}^{n+1}\right)+t_{2 s} I_{m}^{n}\left(1-P_{3}\right)}{\sum_{i=1}^{K} \frac{q\left(\alpha_{i}\right)}{K}\left(\varphi_{4}(h)\right)^{-\alpha_{i}}+\left(d+\varepsilon_{x}\right)+\frac{1}{N^{n}}\left(\alpha_{x x} \beta_{x} I_{x}^{n}\right)} \\
& +\frac{\gamma_{x} I_{x}^{n}-\sum_{i=1}^{K} \frac{q\left(\alpha_{i}\right)}{K} \sum_{j=1}^{n+1} \omega_{j}^{\alpha_{i}} L_{x}^{n+1-j}}{\left(\sum_{i=1}^{K} \frac{q\left(\alpha_{i}\right)}{K}\left(\varphi_{4}(h)\right)^{-\alpha_{i}}+\left(d+\varepsilon_{x}\right)+\frac{1}{N^{n}}\left(\alpha_{x x} \beta_{x} I_{x}^{n}\right)\right.}, \\
I_{s}^{n+1}= & \frac{\varphi_{5}(h) \beta_{s} \frac{I_{s}^{n}}{N^{n}}\left(\alpha_{s s} L_{s}^{n+1}+\left(1-\lambda_{s}\right)\left(S^{n+1}+\sigma_{s} R^{n+1}\right)\right)}{\sum_{i=1}^{K} \frac{q\left(\alpha_{i}\right)}{K}\left(\varphi_{5}(h)\right)^{-\alpha_{i}}+\left(d+\delta_{s}\right)} \\
& +\frac{\left(\gamma_{s}-\left(t_{2 s}\right)\right) I_{s}^{n}+\varepsilon_{s} L_{s}^{n+1}-\sum_{i=1}^{K} \frac{q\left(\alpha_{i}\right)}{K} \sum_{j=1}^{n+1} \omega_{j}^{\alpha_{i}} I_{s}^{n+1-j}}{\sum_{i=1}^{K} \frac{q\left(\alpha_{i}\right)}{K}\left(\varphi_{5}(h)\right)^{-\alpha_{i}}+\left(d+\delta_{s}\right)} \\
I_{m}^{n+1}= & \frac{\beta_{m} \frac{I_{m}^{n}}{N^{n}}\left(\alpha_{m m} L_{m}^{n+1}+\left(1-\lambda_{m}\right)\left(S^{n+1}+\sigma_{m} R^{n+1}+\alpha_{s m} L_{s}^{n+1}\right)\right)}{\sum_{i=1}^{K} \frac{q\left(\alpha_{i}\right)}{K}\left(\varphi_{6}(h)\right)^{-\alpha_{i}}+\left(d+\delta_{m}\right)} \\
& +\frac{\left(\gamma_{m}-\left(t_{2 m}\right)\right) I_{m}^{n}+\varepsilon_{m} L_{m}^{n+1}-\sum_{i=1}^{K} \frac{q\left(\alpha_{i}\right)}{K} \sum_{j=1}^{n+1} \omega_{j}^{\alpha_{i}} I_{m}^{n+1-j}}{\sum_{i=1}^{K} \frac{q\left(\alpha_{i}\right)}{K}\left(\varphi_{6}(h)\right)^{-\alpha_{i}}+\left(d+\delta_{m}\right)} \\
I_{x}^{n+1}= & \frac{\beta_{x} \frac{I_{x}^{n}}{N^{n}}\left(\alpha_{x x} L_{x}^{n+1}+\left(1-\lambda_{x}\right)\left(S^{n+1}+\sigma_{x} R^{n+1}+\alpha_{s x} L_{s}^{n+1}+\alpha_{m x} L_{m}^{n+1}\right)\right)}{\sum_{i=1}^{K} \frac{q\left(\alpha_{i}\right)}{K}\left(\varphi_{7}(h)\right)^{-\alpha_{i}}+\left(d+\delta_{x}\right)} \\
& +\frac{\left(\gamma_{x}-\left(t_{2 x}\right)\right) I_{x}^{n}+\varepsilon_{x} L_{x}^{n+1}-\sum_{i=1}^{K} \frac{q\left(\alpha_{i}\right)}{K} \sum_{j=1}^{n+1} \omega_{j}^{\alpha_{i}} I_{x}^{n+1-j}}{\sum_{i=1}^{K} \frac{q\left(\alpha_{i}\right)}{K}\left(\varphi_{7}(h)\right)^{-\alpha_{i}}+\left(d+\delta_{x}\right)} \\
R^{n+1}= & \frac{t_{1 s} P_{1} L_{s}^{n+1}+P_{2} t_{2 s} I_{s}^{n+1}+t_{2 m} P_{3} I_{m}^{n}+t_{2 x} I_{x}^{n}-\sum_{i=1}^{K} \frac{q\left(\alpha_{i}\right)}{K} \sum_{j=1}^{n+1} \omega_{j}^{\alpha_{i}} R^{n+1-j}}{\sum_{i=1}^{K} \frac{q\left(\alpha_{i}\right)}{K}\left(\varphi_{8}(h)\right)^{-\alpha_{i}}+d+\frac{1}{N^{n}}\left(\sigma_{s} \beta_{s} I_{s}^{n}+\sigma_{m} \beta_{m} I_{m}^{n}+\sigma_{x} \beta_{x} I_{x}^{n}\right)}
\end{aligned} .
$$

\section{Numerical Results and Discussions}

The purpose of this section is to show that NSFD designed in this paper provides good approximations for distributed order fractional differential equations. In all simulations we use the initial conditions $\left(S(0), L_{s}(0)\right.$, $\left.L_{m}(0), L_{x}(0), I_{s}(0), I_{m}(0), I_{x}(0), R(0)\right)=(5000,50,50,50,30,30,30,60)$, with the parameters in Table 1 . The approximate solutions of the proposed system are given in figures 1-5 at different values of $q(\alpha)$. In Fig. 1, the effect of different $q(\alpha)$ on the behavior the approximate solutions for state variables $I_{s}, I_{m}, I_{x}$ and $R$ with comparered the obtianed results with integer case when $q(\alpha)=1$. This figure is given to demonstrate how the distributed order fractional model is a generalization of the integer order model. Fig. 2, shows the behavior of the approximate solutions for state variables $S(t)$ and $I_{x}(t)$ by using NSFDM and SFDM at $h=2$, we can clearly see, NSFDM converge to correct endemic equilibrium but the obtain soluations of SFDM is unstable. Fig. 3, shows the behavior of the approximate solutions of the state variables $I_{m}, I_{x}, R$ at different Dirac delta function by using NSFDM. Fig. 4 show that, the obtianed solutions when $q(\alpha)=\delta(\alpha-0.75)$ are excellent agreement with $q(\alpha)=0.75$. Fig. 5 , shows the relationship between $S(t)$ with $I_{x}(t)$ and $R(t)$ with $I_{m}(t)$ when $q(\alpha)=\delta(\alpha-0.5)$ using NSFDM. For analyzing the stability of the proposed system, we compute $\Upsilon_{n_{A(s)}}(J)$ in the case that the density varies. The results are shown in Table 2. Numerical results are provided to show that NSFD is computationally efficient. From the numerical experiments, we observed that NSFD schemes allow to replicate quite well the behavior of the solution in the classical problems with integer-order. Hence, we can conclude that the developed NSFD schemes are useful for solving distributed order fractional equations. 

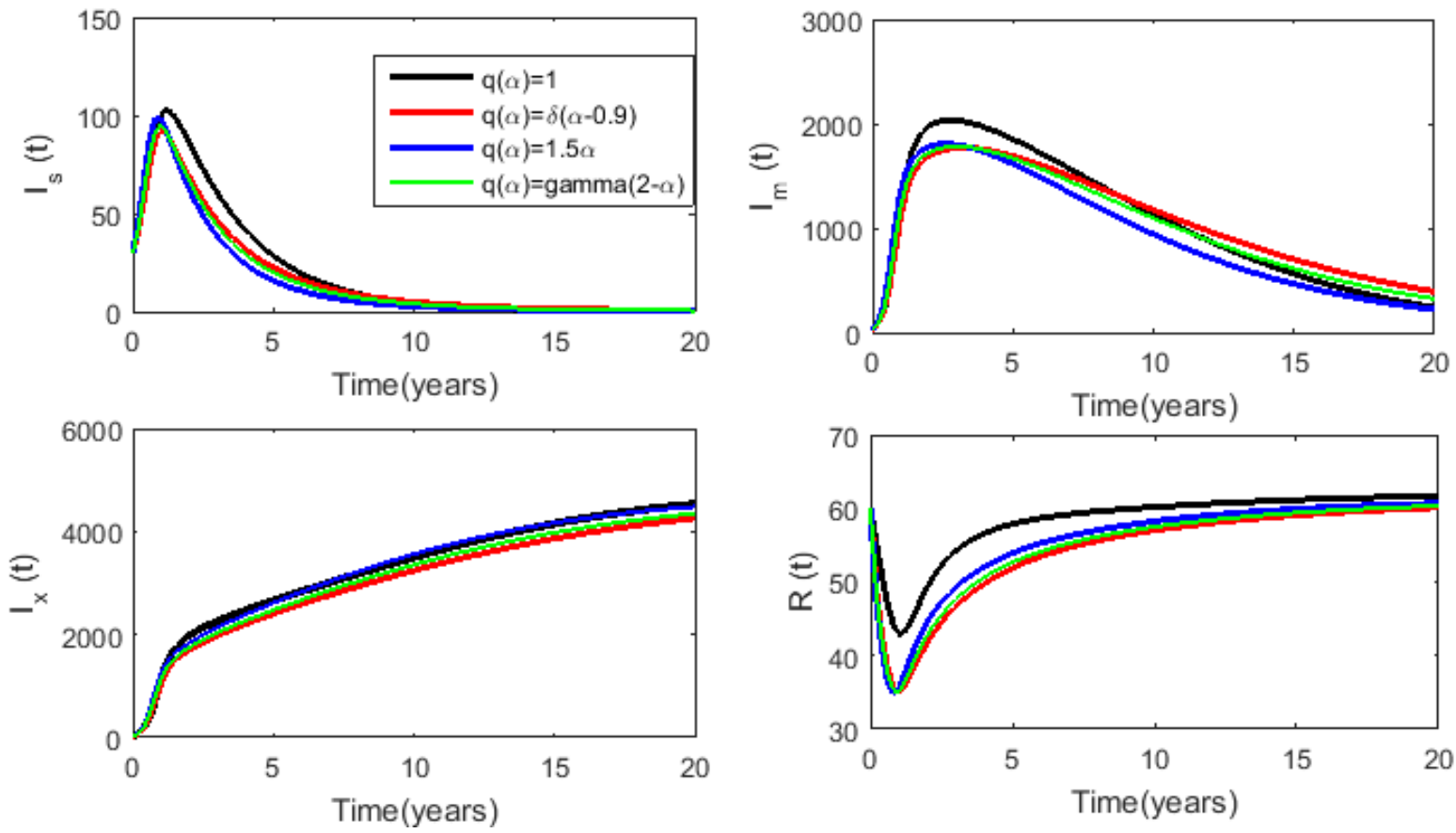

Figure 1. The numerical simulations of $I_{s}, I_{m}, I_{x}, R$ at different $q(\alpha)$ using NSFDM.
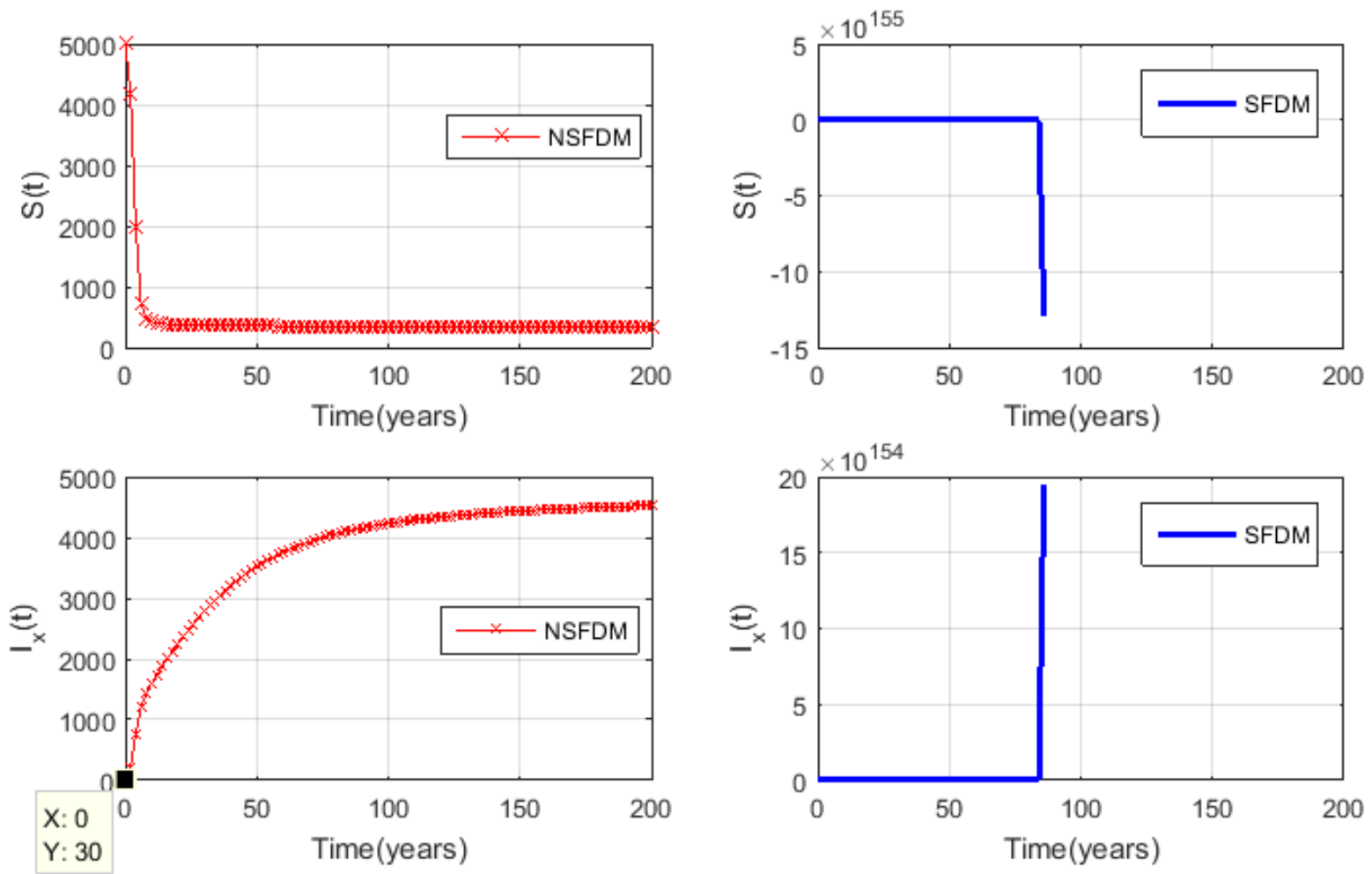

Figure 2. The numerical simulations of $S(t), I_{x}$ at $q(\alpha)=\delta(\alpha-0.6)$ and $h=2$ using NSFDM and SFDM. 

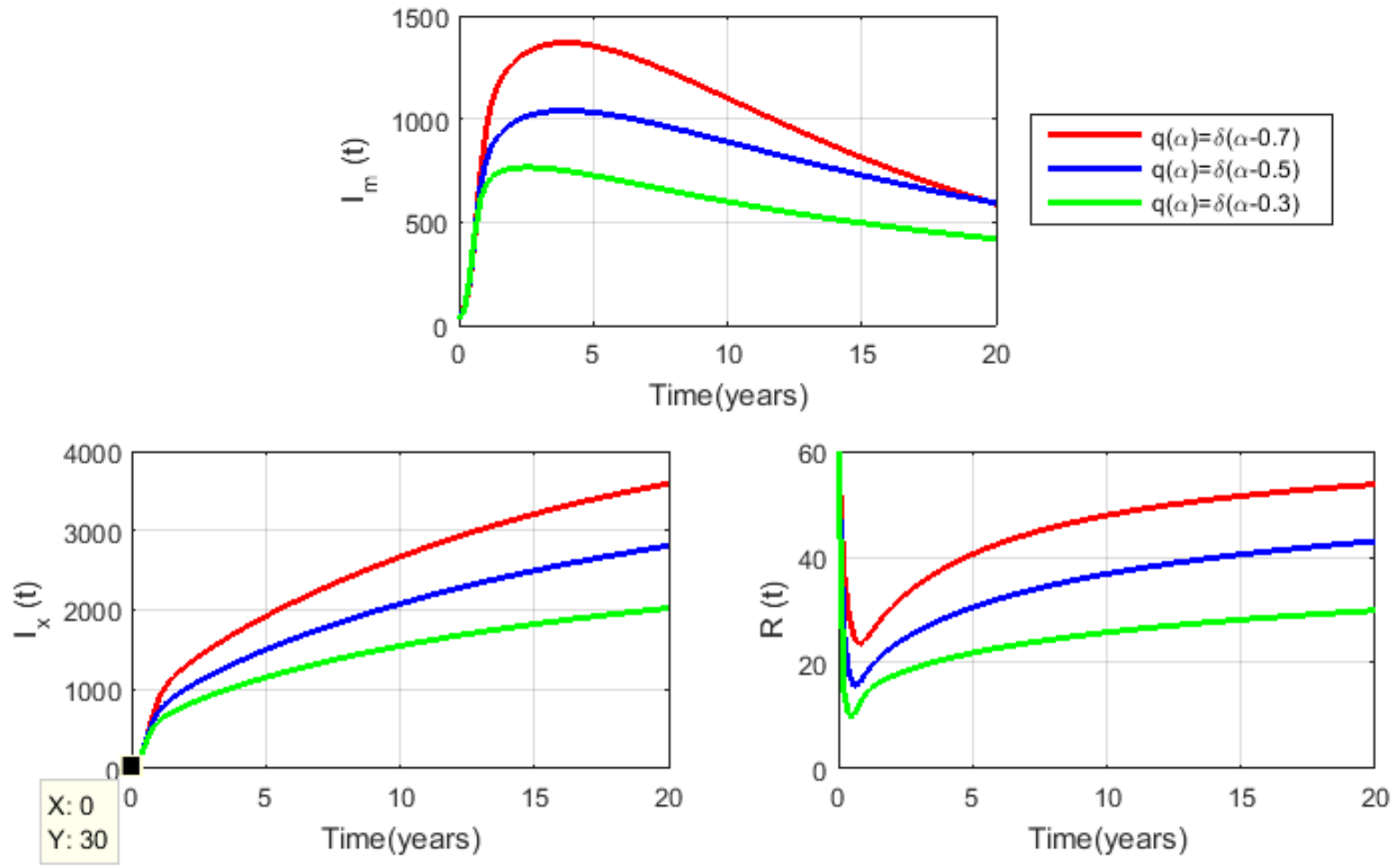

Figure 3. The numerical simulations of $I_{m}, I_{x}, R$ at different values of $q(\alpha)$ and $h=2$ using NSFDM.

Table 2. Stability analysis of system $10-17$ for various density functions.

\begin{tabular}{|c|c|}
\hline$q(\alpha)$ & $\Upsilon_{n_{A(s)}}(J)$ \\
\hline$q(\alpha)=\delta(\alpha-0.7)$ & $\left(352.7115,0.5683,326.5762,1.8557 \times 10^{3}, 1.2735,588.8249,3.5922 \times 10^{3}, 53.7212\right)$ \\
\hline$q(\alpha)=\delta(\alpha-0.8)$ & $\left(349.1680,0.4315,281.8769,1.9830 \times 10^{3}, 1.0480,512.1312,3.9448 \times 10^{3}, 57.5041\right)$ \\
\hline$q(\alpha)=\delta(\alpha-0.9)$ & $\left(345.8485,0.2510,215.4739,2.0867 \times 10^{3}, 0.6521,391.6610,4.2592 \times 10^{3}, 60.1127\right)$ \\
\hline$q(\alpha)=\delta(\alpha-0.95)$ & $\left(344.2361,0.1454,175.8648,2.1297 \times 10^{3}, 0.3890,318.8696,4.4010 \times 10^{3}, 61.0091\right)$ \\
\hline
\end{tabular}

\section{Conclusions}

In this paper, a distributed order fractional multi-strain TB model has been presented as a general model. A nonstandard numerical scheme has been introduced to numerically study the approximate solution of proposed model problem. Some figures are given to demonstrate how the distributed order fractional model is a generalization of the integer and fractional order models where this dynamical model is more suitable to describe the biological phenomena with memory than the integer and fractional order model. The stability of the equilibrium points is investigated. It is concluded that NSFDM can be applied to solve such distributed order differential equations simply and given the large stability regions. 

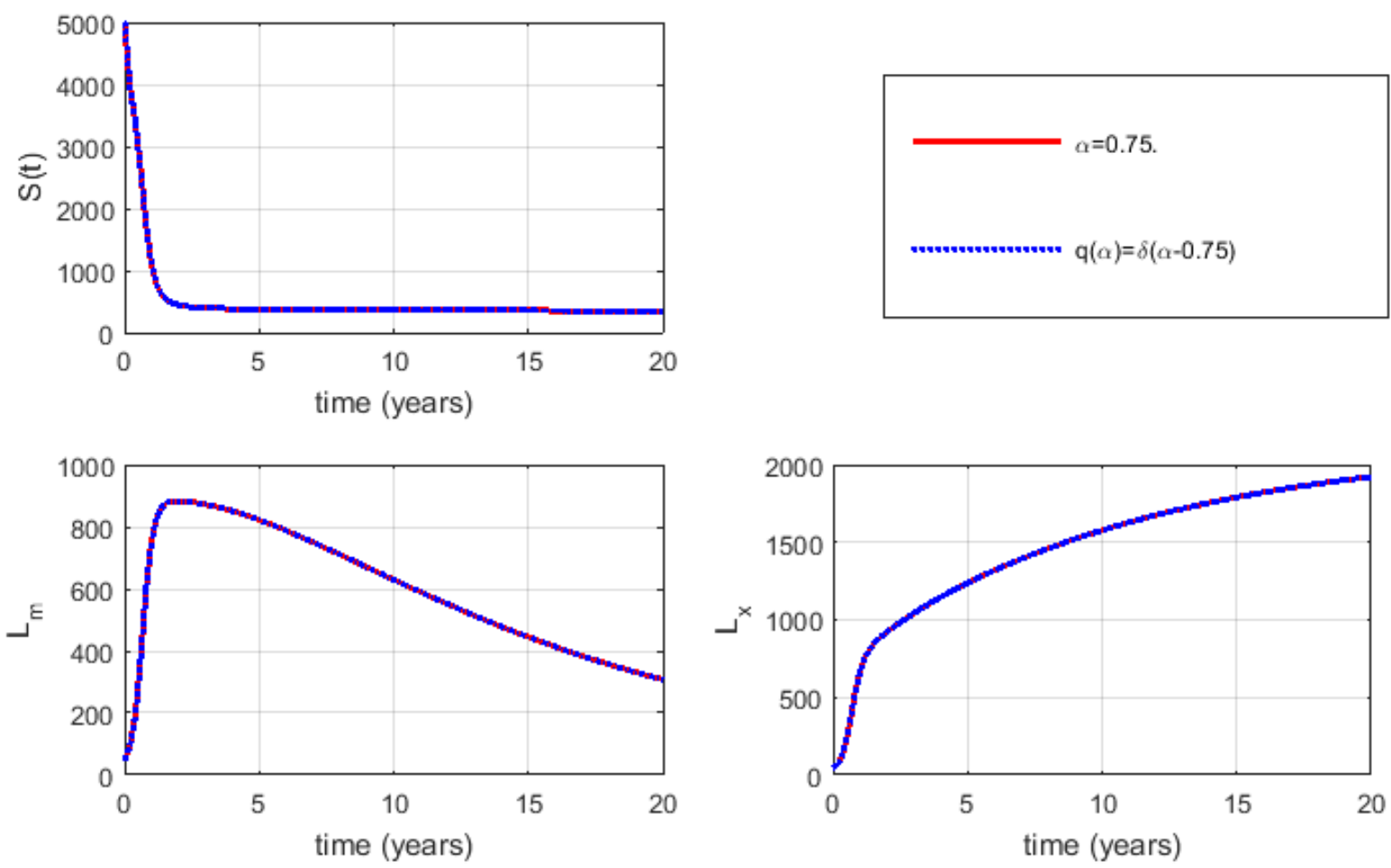

Figure 4. The numerical simulations of $S, L_{m}, L_{x}$ at $q(\alpha)=\delta(\alpha-0.75), q(\alpha)=0.75$ using NSFDM.
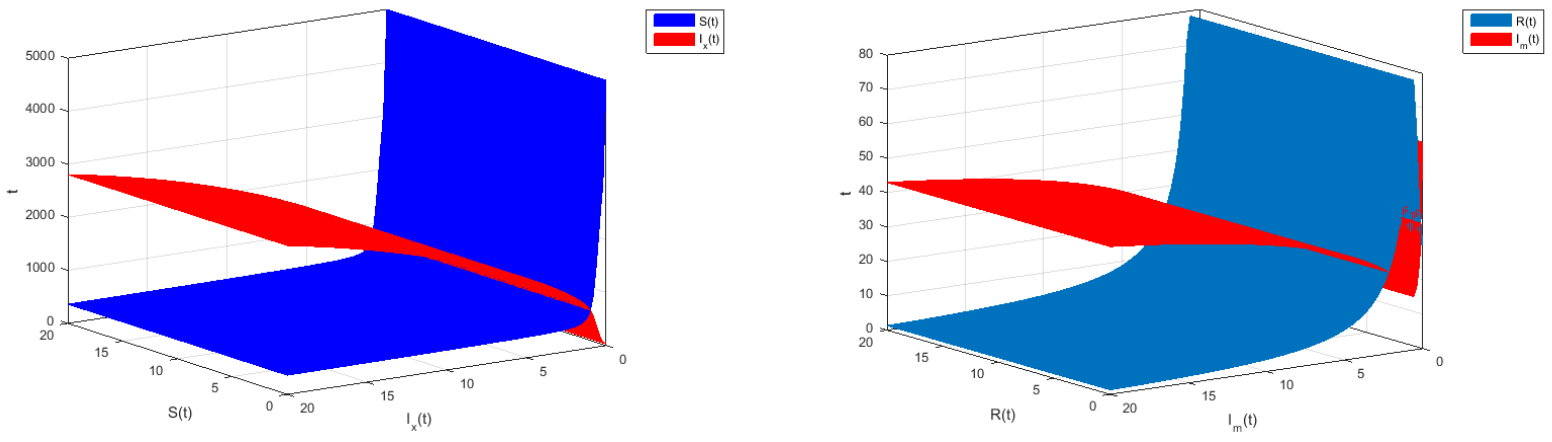

Figure 5. Relation between $S(t)$ with $I_{x}(t)$ and $R(t)$ with $I_{m}(t)$ when $q(\alpha)=\delta(\alpha-0.5)$ using NSFDM.

\section{Acknowledgement}

The authors thank the referees for constructive comments and suggestions which have improved the paper.

\section{Funding}

This research received no specific grant from any funding agency in the public, commercial, or not-for-profit sectors. 
References

1. C.F. Lorenzo, T.T. Hartley, Variable order and distributed order fractional operators. Nonlinear Dynam. 29(1), 57-98, (2002).

2. H.G. Sun, W. Chen, H. Wei, Y. Chen, A comparative study of constant-order and variable-order fractional models in characterizing memory property of systems. Eur. Phys. J. Spec. Top., 193(1), 185-192, (2011).

3. H. Sun, A. Chang, Y. Zhang, W. Chen, A review on variable-order fractional differential equations: Mathematical foundations, physical models, and its applications, Fract. Calc. Appl. Anal., 22(1), 27-59, (2019).

4. R. Almeida, N. R. O. Bastos, M. T. Monteiro, A fractional Malthusian growth model with variable order using an optimization approach, Stat., Optim. Inf. Comput., 6, 4-11, (2018).

5. S. Rosa, D. F. M. Torres, Parameter Estimation, Sensitivity Analysis and Optimal Control of a Periodic Epidemic Model with Application to HRSV in Florida, Stat., Optim. Inf. Comput., 6, 139-149, (2018).

6. J. Arino and I. A. Soliman, A model for the spread of tuberculosis with drug-sensitive and emerging multidrugresistant and extensively drug resistant strains, Mathematical and Computational Modelling, Wiley, 1-120, DOI : $10.1002 / 9781118853887$.ch5, (2015)

7. R. Anguelov and J. M. S. Lubuma, Nonstandard finite difference method by nonlocal approximation, Mathematics and Computers in Simulation, 61(3-6), 465-475, (2003).

8. P. Agarwal, E. Karimov, M. Mamchuev and M. Ruzhansky, On boundary-value problems for a partial differential equation with Caputo and Bessel operators. arXiv:1611.01624.

9. L. Dorciak, Numerical models for simulation the fractional-order control systems, UEF-04-94, The Academy of Sciences, Institute of Experimental Physic,Kosiice, Slovak Republic, (1994).

10. A. A. Kilbas, H. M. Srivastava, and J. J. Trujillo, Theory and application of fractional differential equations, Elsevier, New York, NY, USA, (2006)

11. M. M. Meerschaert and C. Tadjeran, Finite difference approximations for fractional advection-dispersion flow equations, J. Comput. Appl. Math., 172, 65-77, (2003).

12. R. E. Mickens, Nonstandard finite difference models of differential equations, World Scientific, Singapore, (2005).

13. R.E. Mickens, A nonstandard finite-difference scheme for the Lotka-Volterra system, Appl. Numer. Math. 45(2-3), 309-314 (2003).

14. R. E. Mickens, Calculation of denominator functions for nonstandard finite difference schemes for differential equations satisfying a positivity condition, Numerical methods for partial differential equations, 23, 672 - 691, (2007).

15. K. B. Oldham and J. Spanier, The fractional calculus, Acadmic Press, New York, NY, USA, (1974).

16. K. C. Patidar, Nonstandard finite difference methods: recent trends and further developments, Journal of Difference Equations and Applications, 22(6), 817-849, (2016).

17. B. M. Vinagre, Y. Q. Chen, and I. Petras, Two direct Tustin discretization methods for fractional-order differentiator/integrator, Journal of the Franklin Institute, 340(5), 349-362, (2003).

18. M. Caputo, Elasticitae Dissipazione, Zanichelli, Bologna, Italy, (1969).

19. M. Caputo, Mean fractional-order-derivatives differential equations and filters, Annali dell University a di Ferrara, 41(1), 73-84, 1995.

20. M. Caputo, Distributed order differential equations modelling dielectric induction and diffusion, Fractional Calculus and Applied Analysis, 4, 421-442, (2001).

21. L. Bagley and P. J. Torvik, On the existence of the order domain and the solution of distributed order equations, International Journal of Applied Mathematics, 1, 865-882, (2000).

22. K. Diethelm and N. J. Ford, Numerical analysis for distributed order differential equations, Journal of Computational and Applied Mathematics, 225(1), 96-104, (2009).

23. A. Refahi Sheikhani, H. Saberi Nadjafi, A. Ansari, and F. Mehrdoust, Analytic study on linear system of distributedorder fractional differntional equations, Le Matematiche, 67, 313, (2012).

24. H. Saberi Najafi, A. Refahi Sheikhani, and A. Ansari, Stability analysis of distributed order fractional differential equations, Abstract and Applied Analysis, vol. 2011, Article ID 175323, 12 pages, (2011).

25. N. H. Sweilam, S. M. AL-Mekhlafi and T. A. Assiri, Numerical study for time delay multistrain Tuberculosis model of fractional order, Complexity, Volume 2017 (2017), Article ID1047384, 14pages https:// doi .org/10.1155/2017/1047384.

26. N. H. Sweilam, S. M. AL-Mekhlafi, D. Baleanu, Efficient numerical treatments for a fractional optimal control nonlinear tuberculosis model, International Journal of Biomathematics, 11(8), (32 pages), DOI :10.1142/S1793524518501152, (2018).

27. N. H. Sweilam, S. M. AL-Mekhlafi, Shifted Chebyshev spectral collocation method for solving optimal control of fractional multistrain tuberculosis model, Fractional Differential Calculus, 8(1), 1-31, (2018)

28. N. H. Sweilam, S. M. AL-Mekhlafi, Comparative study for multi-strain Tuberculosis (TB) model of fractional order, Journal of Applied Mathematics and Information Sciences, 10(4), 1403-1413, (2016).

29. N. H. Sweilam, S. M. AL-Mekhlafi, Optimal control for a time delay multi-strain tuberculosis fractional model: a numerical approach, IMA Journal of Mathematical Control and Information, 36(1), 317-340, (2019).

30. N. H. Sweilam, I. A. Soliman, S.M. Al-Mekhlafi, Nonstandard finite difference method for solving the multi-strain TB model, Journal of the Egyptian Mathematical Society, 25 (2), 129-138, http://dx. doi.org/10.1016/j.joems.2016.10.004, (2017).

31. N. H. Sweilam, S. M. AL-Mekhlafi, On the optimal control for fractional multi-strain TB model, Optimal Control Applications and Methods 37 (6), 1355-1374, (2016).

32. N. H. Sweilam, S. M. AL-Mekhlafi, Numerical study for multi-strain Tuberculosis (TB) model of variable-order fractional derivatives, Journal of Advanced Research, 7(2), 271-283, (2016).

33. W. H. Organization,Multidrug and extensively drug-resistant TB (M/XDR-TB): 2012 global report on surveillance and response, World Health Organization, (2012). 